\title{
Incidence trends and associated factors of diabetes mellitus in Korean children and adolescents: a retrospective cohort study in Busan and Gyeongnam
}

Jung Hyun Lee, MD, PhD', Yu-Mi Kim, MD, PhD², Min Jung Kwak, $M D, P^{3}$, Su Yung Kim, MD, $\mathrm{PhD}^{4}$, Hyun-Ji Kim, $\mathrm{MD}^{5}$, Chong Kun Cheon, MD, PhD ${ }^{4}$, Woo Yeong Chung, $\mathrm{MD}, \mathrm{PhD}^{6}, \mathrm{Im}$ Jeong Choi, $\mathrm{MD}^{\mathrm{PhD}}{ }^{7}$, Su Young Hong, MD, $\mathrm{PhD}^{8}$, Hee Won Chueh, MD ${ }^{9}$, Jae-Ho Yoo, $M D^{9}$

1Department of Pediatrics, Kosin
University Gospel Hospital, Kosin
University College of Medicine, Busan,
${ }^{2}$ Department of Preventive Medicine,
Dong-A University College of Medicine,
Busan, ${ }^{3}$ Department of Pediatrics, Pusan
National University Hospital, Pusan
National University School of Medicine,
Busan, ${ }^{4}$ Department of Pediatrics, Pusan
National University Children's Hospital,
Pusan National University School of
Medicine, Yangsan, 'Department of
Pediatrics, Wallace Memorial Baptist
Hospital, Busan, 'ㄹepartment of
Pediatrics, Inje University College of
Medicine, Busan, 'Department of
Pediatrics, Mirae Children's Hospital,
Busan, ${ }^{8}$ Department of Pediatrics, ljoeun
Children's Hospital, Busan, ${ }^{9}$ Department
of Pediatrics, Dong-A University College
of Medicine, Busan, Korea

Received: 28 October, 2015

Revised: 7 December, 2015

Accepted: 14 December, 2015

Address for correspondence: Jae-Ho Yoo, MD

Department of Pediatrics, Dong-A University College of Medicine, 32 Daesingongwon-ro, Seo-gu, Busan 49201, Korea

Tel: +82-51-240-2883

Fax: +82-51-242-2765

E-mail: pedendo@dau.ac.kr
Purpose: This study investigated the incidence trends and associated factors of type 1 (T1DM) and type 2 diabetes mellitus (T2DM) in children and adolescents under 15 years of age in Busan and Gyeongnam, Korea from 2001 to 2010.

Methods: Medical records of newly diagnosed diabetes patients ( $n=328 ; 160$ males, 168 females) were collected in questionnaire form from 5 tertiary and 42 general hospitals in Busan and Gyeongnam.

Results: The average crude incidence rate of T1DM and T2DM was 2.01/100,000 (95\% confidence interval [Cl], 1.76-2.28) and 0.75/100,000 (95\% Cl, 0.60-0.92), respectively. The incidence rate ratio (IRR) of T1DM was 1.31 (95\% Cl, 1.01-1.69), and that of T2DM was $1.97(95 \% \mathrm{Cl}, 1.25-3.11)$ in the latter half-decade (2006 to 2010) compared to the early half-decade (2001 to 2005). There were gradually increasing incidence trends in both T1DM and T2DM over the 10-year period ( $P$ for trend: T1DM, 0.0009; T2DM, <0.0001). Age-specific IRR was highest in the 10- to 14-yearold group, regardless of diabetes type. In particular, a rapid increase in incidence of T2DM occurred in the 10- to 14-year-old group. IRR for females was $1.07(95 \% \mathrm{Cl}$, 0.83-1.38) for T1DM and 1.56 (95\% Cl, 1.01-2.41) for T2DM. IRR for Busan (urban) was 1.41 (95\% Cl, 1.09-1.83) for T1DM and 1.49 (95\% Cl, 0.96-2.30) for T2DM.

Conclusion: T1DM and T2DM incidence both increased over time in youth under age 15 living in Busan and Gyeongnam; in particular, the incidence of T2DM in adolescents increased more rapidly.

Keywords: Diabetes, Korea, Incidence, Child, Adolescent

\section{Introduction}

Diabetes mellitus (DM) is a chronic metabolic disease that occurs due to impaired insulin secretion or action, causing defects in carbohydrate, protein, and lipid metabolism ${ }^{1)}$. There are two major types of DM: type 1 diabetes (T1DM) and type 2 (T2DM). T1DM comprises twothirds of all DM occurring among children and adolescents $\mathrm{s}^{2,3)}$.

Recently, an overall change in incidence trends has occurred due to the global increase of T1DM and T2DM among children and adolescents ${ }^{4,5)}$. Incidence differences are highly dependent on ethnic group, region, and environmental factors. For example, China's national incidence rate is 0.1/100,000 versus Finland's 40.9/100,000, which represents a difference of over 350 folds $^{4)}$. Korea and Japan have also demonstrated very low incidence rates ${ }^{6}$. In Korea, a number of nationwide studies into the incidence of T1DM among children and adolescents under the age of 15 years were conducted up until 2000. Korean T1DM incidence rates showed gradual increases from 1.06/100,000 to 1.36/100,000. A study in China covering similar time periods showed an increase in T1DM incidence ${ }^{6}$.

T2DM incidence has increased significantly in parallel with increased youth obesity ${ }^{7.8}$. 
T2DM among children and adolescents tends to occur more frequently in areas with a higher prevalence of diabetic adults ${ }^{8}$. According to the 2005 Korea National Health and Nutrition Examination Survey, T2DM prevalence in adults 20 years old or older increased approximately 5 folds compared to the previous 30 years ${ }^{9}$. Therefore, T2DM incidence is expected to show an increase among Korean children and adolescents as well. However, there has yet been no population-based study of T2DM incidence, and there have been few epidemiological studies on DM among Korean children and adolescents. Therefore, the authors conducted this study to investigate the incidence and associated factors of T1DM and T2DM in children and adolescents younger than 15 years of age in Busan and Gyeongnam, Korea.

\section{Materials and methods}

\section{Subjects}

The study subjects were 328 patients (160 males and 168 females) living in Busan and Gyeongnam, aged 15 years of age or younger. All were newly diagnosed with T1DM or T2DM between January 1,2001, and December 31,2010.

\section{Methods}

One previous study on T1DM incidence in Korea was the precedent case by the Korean Diabetes Association ${ }^{10)}$ and the Korean Pediatric Society ${ }^{11)}$, which distributed questionnaires and analyzed the responses, as well as patient registration information. The present study used the same methodology. Questionnaires were sent to 5 tertiary and 42 general hospitals in Busan and Gyeongnam to request a retrospective survey of medical records (Fig. 1).

The questionnaire was composed of the following items; sex, date of birth, date at the time of diagnosis, address, symptoms and signs, and type of diabetes. Residency in Busan or Gyeongnam was determined by the address used at the time of diagnosis, which was then used to determine regionspecific incidence. The diagnostic criteria for T1DM were the presence of ketoacidosis or fasting serum C-peptide level $\leq 0.6$ $\mathrm{ng} / \mathrm{mL}$; testing positive for anti-islet autoantibodies, even with a C-peptide level $>0.6 \mathrm{ng} / \mathrm{mL}$; and not being able to maintain a blood glucose level without insulin injections for 2 years following diagnosis ${ }^{11)}$. The diagnostic criteria for T2DM were the absence of ketoacidosis, a fasting serum C-peptide level $>0.6 \mathrm{ng} / \mathrm{mL}$, testing negative for anti-islet autoantibodies, or maintaining a blood glucose level without insulin injection for over 2 years following diagnosis.

Positive test results for anti-islet autoantibodies were based on measurements from the insulin autoantibody, the antiglutamic acid decarboxylase antibody, or the islet cell antibody ${ }^{11,12)}$. This study was approved by the Institutional Review Board of Dong-A University Hospital, Busan, Korea (13-107).

\section{Statistical analysis}

Incidence rates were calculated as the numbers of cases per 100,000 persons. The denominator for incidence was based on secondary data from the Korea National Statistical Office ${ }^{13)}$. The 95\% confidence interval (CI) for incidence rate was calculated using a Poisson distribution. The incidence rate was fitted, and a Poisson linear regression model was used to test for a linear trend. To analyze the known risk factors for T1DM development, such as time period, age, sex, region, and seasonality ${ }^{14)}$, a Poisson regression model was applied to calculate the relative incidence rate ratio (IRR). A $P$-value of $<0.05$ was considered statistically significant. Data were analyzed using SAS 9.2 (SAS Inc., Cary, NC, USA).

\section{Results}

\section{Annual and average crude DM incidence rates}

The 10-year average crude T1DM incidence rate for children and adolescents younger than 15 years was 2.01/100,000 (95\% CI, 1.76-2.28), which was 2.68 times greater than the rate of $0.75 /$ 100,000 for T2DM (95\% CI, 0.60-0.92). Annual incidence rates for both T1DM and T2DM gradually increased over time ( $P$ for trend: T1DM, 0.0009; T2DM, $<0.0001$, respectively) (Fig. 2).

Comparisons between the earlier (2001-2005) and latter (2006-2010) half-decades showed that in the earlier halfdecade, T1DM and T2DM incidence rates were 1.80 (95\% CI, $1.48-2.16)$ and 0.45 (95\% CI, 0.30-0.65), while those of the latter half-decade were 2.26 (95\% CI, 1.88-2.70) and 1.09 (95\% CI, 0.84-1.41), respectively (Table 1).

\section{DM incidence rates according to age and sex}

The incidence rates for both T1DM (2.69; 95\% CI, 2.24-3.21)

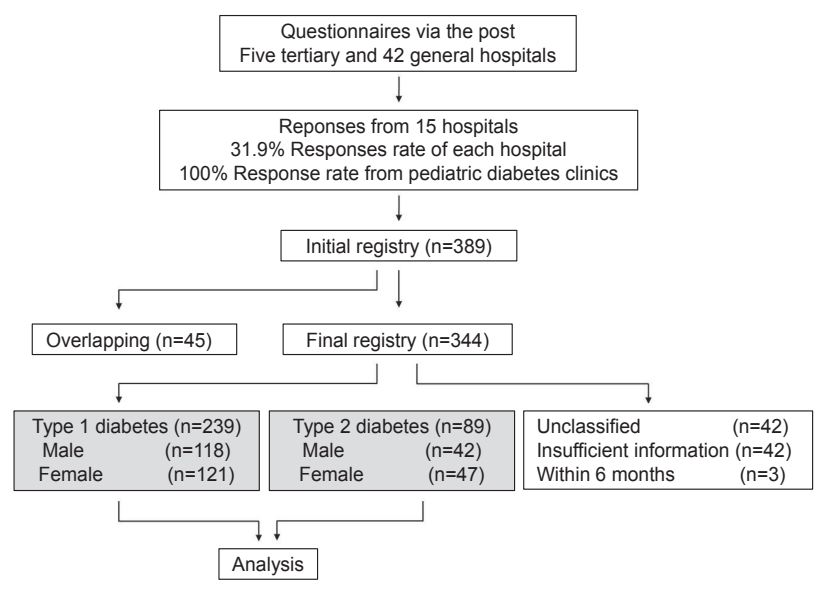

Fig. 1. Procedures: analysis of incidence trends and associated factors of diabetes mellitus in children and adolescents, aged 0-14 years, in Busan and Gyeongnam, Korea (2001-2010). 

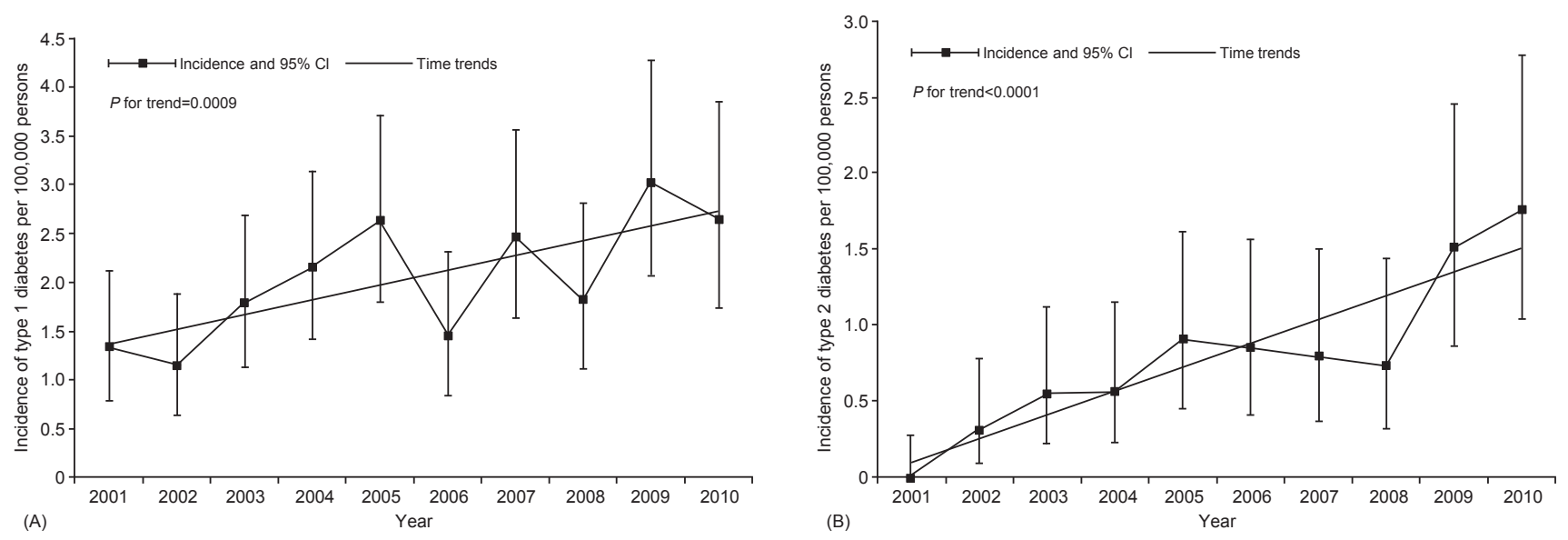

Fig. 2. Incidence of type 1 (A) and type 2 (B) diabetes mellitus in children and adolescents, aged 0-14 years, in Busan and Gyeongnam, Korea (2001-2010). The confidence interval (CI) was calculated using a Poisson distribution. The incidence rate was fitted, and a linear trend test was completed using a Poisson linear regression model.

Table 1. Annual and average crude diabetes mellitus incidence rates in children and adolescents, aged 0-14 years, in Busan and Gyeongnam, Korea (2001-2010)

\begin{tabular}{|c|c|c|c|c|c|}
\hline \multirow{2}{*}{ Year } & \multirow{2}{*}{ Population (n) } & \multicolumn{2}{|c|}{ Type 1 diabetes } & \multicolumn{2}{|c|}{ Type 2 diabetes } \\
\hline & & Cases (n) & Incidence rates ${ }^{\mathrm{a})}\left(95 \% \mathrm{Cl}^{\mathrm{b})}\right)$ & Cases (n) & Incidence rates ${ }^{\mathrm{a}}\left(95 \% \mathrm{Cl}^{\mathrm{b}}\right)$ \\
\hline 2001 & $1,339,760$ & 18 & $1.34(0.80-2.12)$ & 0 & 0 \\
\hline 2002 & $1,311,469$ & 15 & $1.14(0.64-1.89)$ & 4 & $0.31(0.08-0.78)$ \\
\hline 2003 & $1,282,613$ & 23 & $1.79(1.14-2.69)$ & 7 & $0.56(0.22-1.12)$ \\
\hline 2004 & $1,250,423$ & 27 & $2.16(1.42-3.14)$ & 7 & $0.56(0.23-1.15)$ \\
\hline 2005 & $1,215,864$ & 32 & $2.63(1.80-3.72)$ & 11 & $0.90(0.45-1.62)$ \\
\hline 2006 & $1,173,179$ & 17 & $1.45(0.84-2.32)$ & 10 & $0.85(0.41-1.57)$ \\
\hline 2007 & $1,135,231$ & 28 & $2.47(1.64-3.56)$ & 9 & $0.79(0.36-1.50)$ \\
\hline 2008 & $1,095,948$ & 20 & $1.85(1.11-2.82)$ & 7 & $0.64(0.27-1.32)$ \\
\hline 2009 & $1,055,991$ & 32 & $3.03(2.07-4.23)$ & 16 & $1.52(0.87-2.46)$ \\
\hline 2010 & $1,020,462$ & 27 & $2.65(1.74-3.85)$ & 18 & $1.76(1.05-2.79)$ \\
\hline $2001-2005$ & $6,400,129$ & 115 & $1.80(1.48-2.16)$ & 29 & $0.45(0.30-0.65)$ \\
\hline 2006-2010 & $5,480,811$ & 124 & $2.26(1.88-2.70)$ & 60 & $1.09(0.84-1.41)$ \\
\hline $2001-2010$ & $11,880,940$ & 239 & $2.01(1.76-2.28)$ & 89 & $0.75(0.60-0.92)$ \\
\hline
\end{tabular}

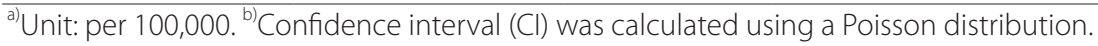

and T2DM $(1.78 ; 95 \%$ CI, 1.41-2.21) were the highest in the 10to 14-year-old group. The T1DM and T2DM incidence rates were 2.17 (95\% CI, 1.80-2.59) and 0.84 (95\% CI, 0.62-1.12) for females and 1.87 (95\% CI, 1.55-2.24) and 0.67 (95\% CI, 0.480.90 ) for males, respectively (Table 2 ). In particular, a recent rapid increase of T2DM incidence was identified among 10-14 year olds ( $P$ for trend: T1DM, 0.0184; T2DM, <0.0001; Fig. 3).

\section{Type 1 and type $2 \mathrm{DM}$ incidence rates according to region and seasonality}

The T1DM and T2DM incidence rates were 2.38 (95\% CI, 2.00-2.82) and 0.93 (95\% CI, 0.70-1.22) in Busan and $1.66(95 \%$ CI, 1.35-2.01) and 0.57 (95\% CI, 0.40-0.80) in Gyeongnam, respectively. T1DM was diagnosed more often during spring (March-May) and winter (December-February), while T2DM was diagnosed more often during spring (March-May) and summer (June-August) (Table 3).

\section{Factors related to DM incidence}

Because the patient population was not large enough to allow us to analyze DM incidence-associated factors by year, the earlier (2001-2005) and latter (2006-2010) half-decades were compared. The adjusted latter half-decade IRRs were 1.31 (95\% CI, 1.01-1.69) and 1.97 (95\% CI, 1.25-3.11) for T1DM and T2DM, respectively.

The age-specific adjusted IRR was highest in the 10- to 14-year-old group for both T1DM (1.56; 95\% CI, 1.09-2.23) and T2DM (2.87; 95\%CI, 1.30-6.35). The sex-specific adjusted IRRs for females were 1.07 (95\% CI, 0.83-1.38) and 1.56 (95\% CI, 1.01-2.41) for T1DM and T2DM, respectively. The regionspecific adjusted IRRs for Busan (an urban area) were 1.41 (95\% CI, 1.09-1.83) and 1.49 (95\% CI, 0.96-2.30) for T1DM and 
Table 2. Diabetes mellitus incidence rates according to sex and age group in children and adolescents, aged 0-14 years, in Busan and Gyeongnam, Korea (2001-2010)

\begin{tabular}{|c|c|c|c|c|c|c|}
\hline \multirow{2}{*}{ Gender } & \multirow{2}{*}{ Age group (yr) } & \multirow{2}{*}{ Population (n) } & \multicolumn{2}{|c|}{ Type 1 diabetes } & \multicolumn{2}{|c|}{ Type 2 diabetes } \\
\hline & & & Case (n) & Incidence rates ${ }^{\mathrm{a})}\left(95 \% \mathrm{Cl}^{\mathrm{b})}\right)$ & Case (n) & Incidence rates ${ }^{\mathrm{a})}\left(95 \% \mathrm{Cl}^{\mathrm{b}}\right)$ \\
\hline \multirow[t]{4}{*}{ Male } & $0-4$ & $1,675,618$ & 19 & $1.13(0.68-1.77)$ & 0 & 0 \\
\hline & $5-9$ & $2,159,179$ & 36 & $1.67(1.17-2.31)$ & 3 & $0.14(0.03-0.40)$ \\
\hline & $10-14$ & $2,473,705$ & 63 & $2.55(1.96-3.29)$ & 39 & $1.58(1.12-2.15)$ \\
\hline & $0-14$ & $6,308,502$ & 118 & $1.87(1.55-2.24)$ & 42 & $0.67(0.48-0.90)$ \\
\hline \multirow[t]{4}{*}{ Female } & $0-4$ & $1,529,125$ & 22 & $1.44(0.90-2.18)$ & 0 & 0 \\
\hline & $5-9$ & $1,906,494$ & 38 & $1.99(1.41-2.74)$ & 4 & $0.21(0.06-0.54)$ \\
\hline & $10-14$ & $2,136,819$ & 61 & $2.85(2.18-3.67)$ & 43 & $2.01(1.46-2.71)$ \\
\hline & $0-14$ & $5,572,438$ & 121 & $2.17(1.80-2.59)$ & 47 & $0.84(0.62-1.12)$ \\
\hline \multirow[t]{4}{*}{ Total } & $0-4$ & $3,204,743$ & 41 & $1.28(0.92-1.74)$ & 0 & 0 \\
\hline & $5-9$ & $4,065,673$ & 74 & $1.82(1.43-2.29)$ & 7 & $0.17(0.07-0.35)$ \\
\hline & $10-14$ & $4,610,524$ & 124 & $2.69(2.24-3.21)$ & 82 & $1.78(1.41-2.21)$ \\
\hline & $0-14$ & $11,880,940$ & 239 & $2.01(1.76-2.28)$ & 89 & $0.75(0.60-0.92)$ \\
\hline
\end{tabular}

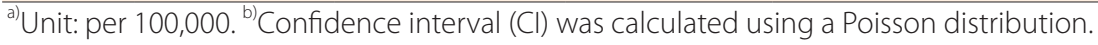

Table 3. Diabetes mellitus incidence rates according to region and season in children and adolescents, aged 0-14 years, in Busan and Gyeongnam, Korea (2001-2010)

\begin{tabular}{|c|c|c|c|c|c|}
\hline \multirow{2}{*}{ Region } & \multirow{2}{*}{ Population (n) } & \multicolumn{2}{|c|}{ Type 1 diabetes } & \multicolumn{2}{|c|}{ Type 2 diabetes } \\
\hline & & Case $(n)$ & Incidence rates ${ }^{\mathrm{a})}\left(95 \% \mathrm{Cl}^{\mathrm{a}}\right)$ & Case $(n)$ & Incidence rates ${ }^{\mathrm{a})}\left(95 \% \mathrm{Cl}^{\mathrm{a})}\right)$ \\
\hline \multicolumn{6}{|l|}{ Region } \\
\hline Busan (urban) & $5,787,727$ & 138 & $2.38(2.00-2.82)$ & 54 & $0.93(0.70-1.22)$ \\
\hline Gyeongnam (rural) & $6,093,213$ & 101 & $1.66(1.35-2.01)$ & 35 & $0.57(0.40-0.80)$ \\
\hline \multicolumn{6}{|l|}{ Seasonc) } \\
\hline Spring & $11,880,940$ & 64 & $0.54(0.41-0.69)$ & 31 & $0.26(0.18-0.37)$ \\
\hline Summer & $11,880,940$ & 45 & $0.38(0.28-0.51)$ & 35 & $0.29(0.20-0.41)$ \\
\hline Autumn & $11,880,940$ & 61 & $0.51(0.39-0.66)$ & 9 & $0.08(0.03-0.14)$ \\
\hline Winter & $11,880,940$ & 69 & $0.58(0.45-0.73)$ & 14 & $0.12(0.06-0.20)$ \\
\hline Total & $11,880,940$ & 239 & $2.01(1.76-2.28)$ & 89 & $0.75(0.60-0.92)$ \\
\hline
\end{tabular}

a) Unit : per 100,000. b) Confidence interval (Cl) was calculated using a Poisson distribution. 'Spring, March-May; Summer, June-August; Fall, September-November; Winter, December-February.

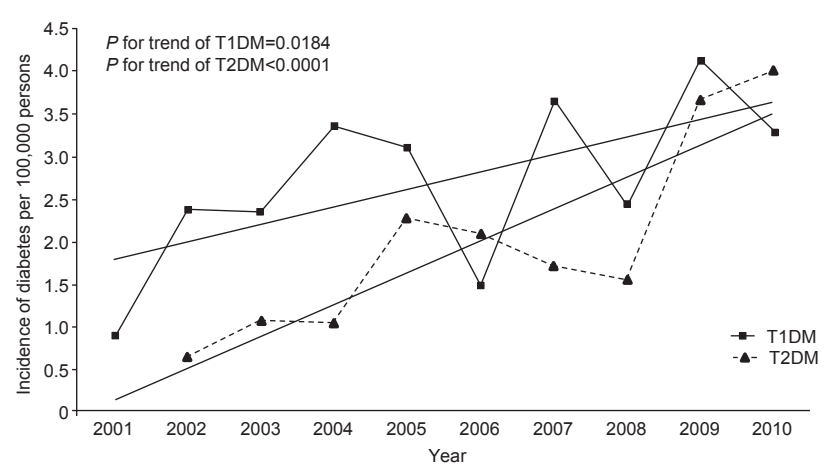

Fig. 3. Diabetes mellitus incidence in children and adolescents, aged 10-14 years, in Busan and Gyeongnam, Korea (2001-2010). T1DM, type 1 diabetes mellitus; T2DM, type 2 diabetes mellitus. The incidence rate was fitted, and a linear trend test was performed with the Poisson linear regression model.

T2DM, respectively. The season-specific adjusted IRRs were not statistically significant (Table 4)

\section{Discussion}

This study investigated the incidence rates and associated factors of T1DM and T2DM in children and adolescents under 15 years of age in Korea's Busan and Gyeongnam provinces from 2001 to 2010.

The T1DM incidences in Busan and Gyeongnam reported here are approximately twice as high as those reported in the Korean Pediatric Society's 1995-2000 Committee for Public Health Statistics of the Korean Pediatric Society report ${ }^{11)}$, which reported an incidence of 1.07/100,000 in Busan and Gyeongnam. T1DM incidence significantly increased in the latter half-decade, and this trend is believed to be nationwide.

The T1DM incidence increase may also have been a result of the higher response rates to the questionnaires in this study compared to those of previous studies ${ }^{10,11)}$. One such study ${ }^{11)}$ showed a response rate of $24 \%$ from each hospital, while the present study showed a rate of $32 \%$. The increased response rates were believed to be a result of the increased utilization of diabetes clinics specializing in child and adolescent T1DM in Busan and Gyeongnam. In fact, diabetes clinics for children and adolescents with pediatric endocrinologists on staff had a 
Table 4. Risk factors related to diabetes mellitus development in children and adolescents, aged 0-14 years, in Busan and Gyeongnam, Korea (2001-2010)

\begin{tabular}{|c|c|c|c|c|c|c|}
\hline \multirow{2}{*}{ Factor } & \multicolumn{3}{|c|}{ Type 1 diabetes } & \multicolumn{3}{|c|}{ Type 2 diabetes } \\
\hline & $\mathrm{RR}^{\mathrm{a})}$ & $95 \% \mathrm{Cl}^{\mathrm{b})}$ & $P$-value & $\mathrm{RR}^{\mathrm{a})}$ & $95 \% \mathrm{Cl}^{\mathrm{b})}$ & $P$-value \\
\hline \multicolumn{7}{|l|}{ Period (yr) } \\
\hline 2001-2005 & 1.00 & & & 1.00 & & \\
\hline 2006-2010 & 1.31 & $1.01-1.69$ & 0.038 & 1.97 & $1.25-3.11$ & 0.004 \\
\hline \multicolumn{7}{|l|}{ Age group (yr) } \\
\hline $0-4$ & 1.00 & & & & & \\
\hline $5-9$ & 1.28 & $0.87-1.88$ & 0.214 & 1.00 & & \\
\hline $10-14$ & 1.56 & $1.09-2.23$ & 0.015 & 2.87 & $1.30-6.35$ & 0.009 \\
\hline \multicolumn{7}{|l|}{ Sex } \\
\hline Male & 1.00 & & & 1.00 & & \\
\hline Female & 1.07 & $0.83-1.38$ & 0.619 & 1.56 & $1.01-2.41$ & 0.044 \\
\hline \multicolumn{7}{|l|}{ Region } \\
\hline Gyeongnam & 1.00 & & & 1.00 & & \\
\hline Busan & 1.41 & $1.09-1.83$ & 0.009 & 1.49 & $0.96-2.30$ & 0.073 \\
\hline \multicolumn{7}{|c|}{ Season of onset ${ }^{c}$} \\
\hline Spring & 1.00 & & & 1.00 & & \\
\hline Summer & 0.69 & $0.47-1.02$ & 0.061 & 0.96 & $0.59-1.56$ & 0.860 \\
\hline Autumn & 0.88 & $0.62-1.25$ & 0.477 & 0.72 & $0.34-1.53$ & 0.392 \\
\hline Winter & 0.98 & $0.70-1.38$ & 0.930 & 0.52 & $0.28-0.99$ & 0.107 \\
\hline
\end{tabular}

${ }^{a)}$ Adjusted rate ratio (RR) for each factor was estimated by a multiple Poisson regression model. ${ }^{\text {b) }}$ Confidence interval (Cl) was calculated using a Poisson distribution. 'Spring, March-May; Summer, June-August; Fall, September-November; Winter, December-February.

$100 \%$ response rate to the questionnaire, and their responses accounted for $77.6 \%$ of the total. This higher response rates may also be accounted for by sampling error, which is a limitation of the current study. The incidences were estimated based on reports from specialists, as was the case with many previous studies $^{11)}$. However, clinical symptoms of T1DM that occur in children and adolescents younger than 15 years of age show a pattern of acute illness ${ }^{10)}$. Therefore, considering the fact that the patients were diagnosed and treated mostly by pediatricians within Busan and Gyeongnam, the sampling error in the present study is thought to be smaller than that of previous studies.

Furthermore, T2DM in children and adolescents is rapidly increasing globally. Before the 1990s, T2DM accounted for fewer than 3\% of all newly diagnosed DM cases in the United States, and this increased to $45 \%$ in the $2000 \mathrm{~s}^{8)}$. In Japan, T2DM incidence from 1975 to 2000 was 3.0/100,000, which was almost double the T1DM incidence ${ }^{15)}$. In Korea, one study examining patients from a single hospital indicated no T2DM patients before 1990, but there was an increased prevalence after $2000^{16)}$. The present study found similar results, in that there was no incidence of T2DM case in 2001, but there were increases in reports of T2DM patients each year after 2002. Much like that of T1DM, T2DM incidence showed a significant increase in the latter half-decade.

There may be several reasons for the T2DM incidence increase. First, the prevalence of obesity in children and adolescents and T2DM prevalence are closely related. Family history of DM, ethnic group genetic factors, industrial development, and westernization of lifestyles and dietary habits all contribute to obesity and T2DM incidences ${ }^{7,8)}$. In Korean studies that examined child and adolescent T2DM patients, Park et al. ${ }^{16)}$ and Yun et al. ${ }^{17)}$ reported that $68 \%$ and $66.6 \%$ of the patients, respectively, were overweight or obese. Second, an increase in the survival rates of groups at high risk for developing T2DM, such as prematurity or intrauterine growth retardation, may contribute to increased T2DM incidence ${ }^{18,19)}$. Third, the utilization of a urine sugar test in school health examinations played an important role in diagnosing T2DM by detecting asymptomatic glycosuria. In studies of child and adolescent T2DM patients in Korea, Hong et al. ${ }^{20)}$ and Yun et al. ${ }^{17)}$ reported that $46.7 \%$ and $47.8 \%$, respectively, of those patients were diagnosed with asymptomatic glycosuria. In Japan, 6.27/100,000 of type 2 DM cases have been diagnosed annually since 1974 through school urine sugar tests ${ }^{15)}$. Another factor is that physicians used to preferentially diagnose DM in pediatric patients as T1DM however, within the past 10 years, these physicians have started to increasingly examine the possibility of T2DM before making a final diagnosis.

However, it is possible that T2DM incidence in children and adolescents younger than 15 was underestimated in the present study because patients with T2DM detected from asymptomatic glycosuria may not actually visit a hospital; alternatively, they may also be treated according to an adult T2DM treatment protocol outside a pediatric department or receiving care in primary health care. Hence, additional studies based on data from the Insurance Review and Assessment Service or an investigation into the frequency of final DM confirmation from urine sugar tests during school health examinations are needed. Because T2DM is three or four times more prevalent than T1DM, greater T2DM awareness is needed in Korea. 
Generally, T1DM occurs most frequently among 10-14 year olds, who are starting to undergo puberty ${ }^{21)}$. Studies by the Korean Diabetes Society ${ }^{10)}$ and the Korean Pediatric Society ${ }^{11)}$ showed that this same age group had the highest T1DM agespecific incidence, and the present study confirms these results. Zhang et al. ${ }^{6}$ reported that the IRR of the 10- to 14-year-old group was about 4 times higher than that of the 0 - to 4 -year-old group. T2DM in children and adolescents, similarly to T1DM, is known to be most prevalent during puberty ${ }^{22}$. Park et al. $\left.{ }^{16}\right)$ reported that the 10- to 14-year-old group showed the highest incidence rate among T2DM patients, and the present study confirms these results.

In the present study, T1DM incidence was 2.68 times higher than that of T2DM. However, when annual incidence trends of T1DM and T2DM in the high-prevalence 10- to 14-yearold group were compared, T2DM showed a rapidly increasing recent trend, and it occurred more frequently than did T1DM in 10- to 14-year-old adolescents in 2010. Although data from a single year cannot be used to surmise that T2DM will be more prevalent than T1DM in the future, T2DM incidence should be more closely monitored.

T1DM incidence in patients under 15 years old varies greatly depending on regional or ethnic factors ${ }^{4}$. In Korea, where ethnic differences are almost nonexistent, regional differences were prominent. In a previous survey, incidence rates outside and inside Seoul were $0.68 / 100,000$ and $1.86 / 100,000$, respectively ${ }^{10)}$. The present study also shows regional differences between the Busan (urban) and Gyeongnam (rural) regions. Urban regions may be more susceptible to the spread of pathogens within the environment with a more dense population, and various environmental factors associated with higher quality of living in terms of nutrition and lifestyle in urban regions may also play a role in the differences ${ }^{6}$. Region-specific T2DM incidence was also higher in Busan than in Gyeongnam. Thus, urbanization and subsequent lifestyle westernization, along with genetic factors, may be possible causes of the increase in T2DM incidence among Asians ${ }^{233}$.

In conclusion, the present study represents the most recent investigations into the changing trends in T1DM and T2DM incidence among Korean children and adolescents younger than 15 during the 2000s. Between 2001 and 2010, T1DM and T2DM incidence rates both showed increasing trends, and T2DM's incidence appears to be increasing at a much faster pace, which may explain the higher incidence of T2DM compared to T1DM among 10-14 year olds in 2010. These results suggest the need for close monitoring of DM incidences in children and adolescents, as well as for follow-up studies concerning T2DM risk factors.

\section{Conflict of interest}

No potential conflict of interest relevant to this article was reported.

\section{Acknowledgments}

The authors wish to express special thanks to the following: Jae Sun Park (Department of Pediatrics, Kosin University College of Medicine); Kyunglae Cho (Department of Pediatrics, Sungkyunkwan University College of Medicine, Masan Samsung Hospital); Eun Sil Park (Department of Pediatrics, Gyeongsang National University College of Medicine); Sun Hee Lee (Department of Pediatrics, Inje University College of Medicine); Eun-Young Kwon (Department of Pediatrics, Good Samsun Hospital); Kyun Woo Lee (Department of Pediatrics, Dae Dong Hospital); So Eun Jun (Department of Pediatrics, Pusan National University School of Medicine); Woo Sik Jung (Department of Pediatrics, Dae Dong Hospital); Yun-Jin Bae (Department of Pediatrics, Pusan Adventist Hospital); and EunJeong Choi (Department of Pediatrics, Ilsin Christian Hospital). The authors also wish to extend their gratitude to the many pediatric physicians in Busan and Gyeongnam who provided valuable data and feedback.

This study was supported by the Dong-A University Research Fund.

\section{References}

1. Korean Society of Pediatric Endocrinology. Pediatric endocrinology. 2nd ed. Seoul: Kwang Moon Publishing Co., 2004.

2. Fagot-Campagna A, Pettitt DJ, Engelgau MM, Burrows NR, Geiss LS, Valdez R, et al. Type 2 diabetes among North American children and adolescents: an epidemiologic review and a public health perspective.J Pediatr 2000;136:664-72.

3. SEARCH for Diabetes in Youth Study Group, Liese AD, D'Agostino RB Jr, Hamman RF, Kilgo PD, Lawrence JM, et al. The burden of diabetes mellitus among US youth: prevalence estimates from the SEARCH for Diabetes in Youth Study. Pediatrics 2006;118:1510-8.

4. DIAMOND Project Group. Incidence and trends of childhood type 1 diabetes worldwide 1990-1999. Diabet Med 2006;23:857-66.

5. Writing Group for the SEARCH for Diabetes in Youth Study Group, Dabelea D, Bell RA, D'Agostino RB Jr, Imperatore $\mathrm{G}$, Johansen JM, et al. Incidence of diabetes in youth in the United States. JAMA 2007;297:2716-24.

6. Zhang H, Xia W, Yu Q, Wang B, Chen S, Wang Z, et al. Increasing incidence of type 1 diabetes in children aged 0-14 years in Harbin, China (1990-2000). Prim Care Diabetes 2008;2:121-6.

7. Kitagawa T, Owada M, Urakami T, Yamauchi K. Increased incidence of non-insulin dependent diabetes mellitus among Japanese schoolchildren correlates with an increased intake of animal protein and fat. Clin Pediatr (Phila) 1998;37:111-5.

8. Pinhas-Hamiel O, Zeitler P. The global spread of type 2 diabetes mellitus in children and adolescents. J Pediatr 
2005;146:693-700.

9. Ko SH, Cha BY. Diabetic peripheral neuropathy in type 2 diabetes mellitus in Korea. Diabetes Metab J 2012;36:6-12.

10. Rhee BD. Epidemiological characteristics of diabetes mellitus among Korean population. J Korean Diabetes Assoc 2003;27:173-8.

11. The Committee for Public Health Statistics. Incidence of type 1 diabetes mellitus in Korea 1995-2000. In: Program and abstract, the 51st Annual Fall Meeting of Korean Pediatric Society; 2001 Oct 19-20, Seoul. Seoul: The Korean Pediatric Society, 2001.

12. American Diabetes Association. Diagnosis and classification of diabetes mellitus. Diabetes Care 2010;33(Suppl 1):S62-9.

13. Korean Statistical Information Service. Statistics of residence registration population [Internet]. Daejeon: Korean Statistical Information Service; 2015 [cited 2012 Jan 17]. Available from: http://kosis.kr/statHtml/statHtml.do?orgId =101\&tblId=DT_1B04005\&conn_path=I2.

14. Maahs DM, West NA, Lawrence JM, Mayer-Davis EJ. Epidemiology of type 1 diabetes. Endocrinol Metab Clin North Am 2010;39:481-97.

15. Urakami T, Morimoto S, Nitadori Y, Harada K, Owada M, Kitagawa T. Urine glucose screening program at schools in Japan to detect children with diabetes and its outcomeincidence and clinical characteristics of childhood type 2 diabetes in Japan. Pediatr Res 2007;61:141-5.
16. Park JM, Yoo EG, Kim DH. Type 2 diabetes mellitus in children. J Korean Pediatr Soc 2002;45:646-53.

17. Yun KA, Lee YA, Shin CH, Yang SW. Clinical course of childhood and adolescence onset type 2 diabetes mellitus. J Korean Soc Pediatr Endocrinol 2009; 14:19-24.

18. Barker DJ, Hales CN, Fall CH, Osmond C, Phipps K, Clark PM. Type 2 (non-insulin-dependent) diabetes mellitus, hypertension and hyperlipidaemia (syndrome $\mathrm{X}$ ): relation to reduced fetal growth. Diabetologia 1993;36:62-7.

19. Mericq V. Prematurity and insulin sensitivity. Horm Res 2006;65(Suppl 3):131-6.

20. Hong EH, Park JS, Lee HS, Cho MH, Ko CW. Clinical characteristics and laboratory findings of children who were newly diagnosed with diabetes mellitus (from 2001 to 2008). J Korean Soc Pediatr Endocrinol 2009;14:110-5.

21. Pundziute-Lycka A, Dahlquist G, Nystrom L, Arnqvist H, Bjork E, Blohme G, et al. The incidence of Type I diabetes has not increased but shifted to a younger age at diagnosis in the 0-34 years group in Sweden 1983-1998. Diabetologia 2002;45:783-91.

22. Pinhas-Hamiel O, Dolan LM, Daniels SR, Standiford D, Khoury PR, Zeitler P. Increased incidence of non-insulindependent diabetes mellitus among adolescents. J Pediatr 1996;128(5 Pt 1):608-15.

23. Lee HY, Won JC, Kang YJ, Yoon SH, Choi EO, Bae JY, et al. Type 2 diabetes in urban and rural districts in Korea: factors associated with prevalence difference. J Korean Med Sci 2010;25:1777-83. 